\title{
The Relationship between the HATCH Score, Neutrophil to Lymphocyte Ratio and Postoperative Atrial Fibrillation After Off-Pump Coronary Artery Bypass Graft Surgery
}

\author{
Burak Erdolu, MD,${ }^{1}$ Ahmet Kagan As, MD,${ }^{1}$ Mesut Engin M, MD ${ }^{2}$ \\ ${ }^{1}$ Department of Cardiovascular Surgery, University of Health Sciences, Bursa Yuksek Ihtisas Training and Research Hospital, Yıldırım/ \\ Bursa, Turkey; ${ }^{2}$ Department of Cardiovascular Surgery, University of Health Sciences, Mehmet Akif İnan Training and Research \\ Hospital, Şanlıurfa, Turkey
}

\section{ABSTRACT}

Backround: Postoperative atrial fibrillation (PoAF), the most common arrythmia observed in $18-40 \%$ of patients following coronary artery bypass surgery, may cause hemodynamic disturbances and increase embolism risk. The aim of this study was to investigate the relationship of HATCH score with PoAF in patients who underwent off-pump coronary artery bypass grafting (OPCABG) and evaluate the effect of preoperatively calculated neutrophil-to-lymphocyte ratio (NLR) on PoAF.

Methods: Patients who underwent OPCABG between January 2014 and January 2019 were included in the study. Preoperative and postoperative data retrospectively were obtained. Patients who did not develop PoAF during the postoperative hospitalization period constituted Group 1; those who did were classified as Group 2.

Results: Ninety-seven patients $(69$ males and 28 females) with a mean age of $54.4 \pm 11.1$ years constituted Group 1, and 26 patients (17 males and 9 females) with a mean age of $61 \pm 12.6$ years constituted Group 2. Significant differences were observed between the 2 groups, in terms of age and HATCH scores $(P=.025$ and $P<.001$, respectively). NLR, number of distal anastomoses, intensive care unit (ICU) stay times, and total hospitalization times were significantly higher in Group $2(P=.021, P=.021, P<$ $.001, P<.001$, respectively). HATCH score was identified as an independent predictor of AF development following OPCABG surgery (OR: 2.125, 95 \% CI: 1.296-3.482, $P=.003)$.

Conclusion: In light of our study, HATCH scores of all patients preoperatively may be calculated so that preventive precautions are taken for high-risk patients.

Received October 14, 2019; accepted December 5, 2019.

Correspondence: Mesut Engin, MD, University of Health Sciences, Mehmet Akif Inan Training and Research Hospital, Department of Cardiovascular Surgery, Esentepe Town, Ertuğrul Street, Karaköprü/Şanluurfa, Turkey, 63200;+904143186000; fax:+904143186707 (e-mail:mesut_kvc_cor@ botmail.com).

\section{INTRODUCTION}

Postoperative atrial fibrillation (PoAF), the most common arrythmia observed in 18-40\% of patients following coronary artery bypass surgery [El-Chami 2010; Ozturk 2019], may cause hemodynamic disturbances and increase embolism risk. Off-pump coronary artery bypass graft (OPCABG) operation protects patients from possible risks of extracorporeal circulation and shortens hospitalization times. PoAF, which may occur following these operations, may increase the morbidity and mortality of the patients and extend hospital stays. Therefore, it is extremely important to determine the risky patient groups during the preoperative period and take the necessary precautions.

Inflammatory parameters have been the subject of research, regarding the results of operations in cardiac surgery, just as in many areas of medicine, and exert various effects on cardiovascular diseases [Aviles 2003]. Neutrophil-to-lymphocyte

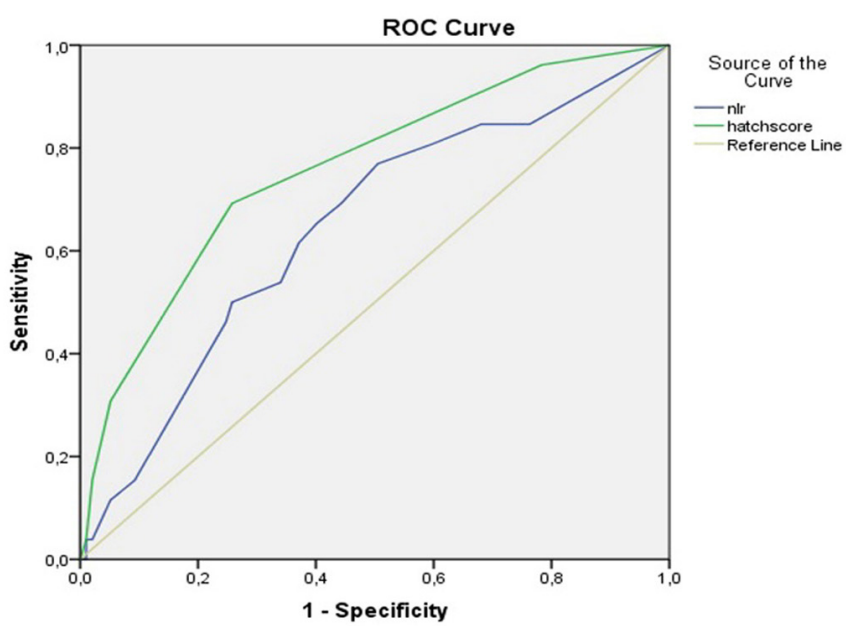

Receiver operation characteristic (ROC) curve and area under the curve (AUC) for NLR and the HATCH score for predicting PoAF. (HATCH score: AUC: $0.759, P<.001,69 \%$ sensitivity and $53 \%$ specificity) (NLR: AUC: $0.644, P=.024,65 \%$ sensitivity and $38 \%$ specificity). A moderate positive correlation was detected between NLR and the HATCH score $(\mathrm{R}=0.427, P<.001)$. 
Table 1. The HATCH score parameters

\begin{tabular}{ll}
\hline Variables & Point \\
\hline Ejection fraction $\leq 40$ & 2 \\
Transischemic attack/stroke & 2 \\
Age $>75$ & 1 \\
Hypertension & 1 \\
Chronic obstructive pulmonary disease & 1
\end{tabular}

ratio (NLR) is one of the most easily obtained parameters, which was found to be associated with worse outcomes in cardiovascular diseases [Papa 2008]. It also has been reported that preoperatively calculated NLR may be associated with PoAF [Gibson 2007].

The HATCH score is a scoring system containing 5 different variables that play a role in the etiology of atrial fibrillation (AF) (Table 1). It is used in cardiology clinics to demonstrate $\mathrm{AF}$ recurrence after radiofrequency ablation and shown to predict the development of PoAF after CABG operations with cardiopulmonary bypass (CPB) [Miao 2012; Selvi 2017].

The aim of this study was to investigate the relationship of HATCH score with PoAF in patients who underwent OPCABG, evaluate the effect of preoperatively calculated NLR on PoAF and its correlation with the HATCH score.

\section{MATERIALS AND METHODS}

Patients who underwent OPCABG between January 2014 and January 2019 in Health Sciences University Bursa High Specialty Educational and Research Hospital were included in this retrospective study. Approval from the local ethics committee was obtained. The study was conducted in accordance with the principles of the Declaration of Helsinki. Preoperative (demographic characteristics, history of comorbid diseases, ejection fraction (EF), and blood parameters) and postoperative (intensive care stay, total duration of hospitalization, total drainage, and PoAF development status) data were obtained from the hospital registry and patient files. Excluded from the study were patients who preoperatively were treated with amiodarone, those with a history of $\mathrm{AF}$, moderate or higher mitral regurgitation or known systemic inflammatory disease, those who underwent emergency operations or reoperations, and chronic hemodialysis patients. After the implementation of exclusion criteria, 123 patients who consecutively were operated were included in the study. The HATCH score of each patient was calculated from the recorded clinical parameters.

Blood parameters of the patients were measured from blood samples obtained from antecubital veins during hospitalization. Whole blood counts were performed with an automated whole blood analyzer, and biochemical values were measured with an automatic device. Hemoglobin, hematocrit, neutrophil, lymphocyte, platelet and white blood cell counts, blood urea nitrogen, creatinine, and
Table 2. Preoperative valuables and demographic datas of the patients

\begin{tabular}{lccc}
\hline & $\begin{array}{c}\text { Group 1 } \\
(\mathrm{N}=97)\end{array}$ & $\begin{array}{c}\text { Group 2 } \\
(\mathrm{N}=26)\end{array}$ & $P$ \\
\hline Age(years) (mean \pm sd) & $54.4 \pm 11.1$ & $61 \pm 12.6$ & .025 \\
Female gender, N (\%) & $28(28.8 \%)$ & $9(34.6 \%)$ & .570 \\
Previous PCl, N (\%) & $11(11.3 \%)$ & $4(15.3 \%)$ & .576 \\
Hypertension, N (\%) & $53(54.6 \%)$ & $19(65.5 \%)$ & .141 \\
Hyperlipidemia, N (\%) & $38(39.1 \%)$ & $9(34.6 \%)$ & .432 \\
COPD, N (\%) & $13(13.4 \%)$ & $6(23 \%)$ & .094 \\
BMI (kg/m²) (mean \pm sd) & $26.9 \pm 3.9$ & $27.3 \pm 4.2$ & .286 \\
Diabetes mellitus, N (\%) & $19(19.5 \%)$ & $7(26.9 \%)$ & .324 \\
Current smoker, N (\%) & $23(23.7 \%)$ & $5(19.2 \%)$ & .694 \\
Beta-blocker use, N (\%) & $21(21.6 \%)$ & $6(23 \%)$ & .744 \\
ACE-l/ ARB use, N (\%) & $14(14.4 \%)$ & $4(15.3 \%)$ & .856 \\
Ejection fraction, (\%) (mean \pm sd) & $46.8 \pm 9.3$ & $45.2 \pm 10.7$ & .761 \\
Left atrial diameter, (mean \pm sd) & $35.9 \pm 4.1$ & $36.7 \pm 3.9$ & .447 \\
HATCH score, (mean \pm sd) & $2.1 \pm 0.9$ & $3 \pm 1.2$ & $<.001$ \\
\hline
\end{tabular}

ACE-I: angiotensin-converting enzyme inhibitor, ARB: angiotensin-receptor blocker, BMI: body mass index, COPD: chronic obstructive pulmonary disease, $\mathrm{PCl}$ : percutaneus coronary intervention

C-reactive protein (CRP) values were recorded and NLR values were calculated.

All patients postoperatively were followed up in the intensive care unit. In this process, electrocardiography (ECG) monitoring continuously was performed. In the inpatient clinic, where patients were admitted to on the second postoperative day, 12-lead ECGs of patients, who complained of palpitation, dizziness or dyspnea, immediately were evaluated. The irregular rhythm, which continued for 2 minutes with no distinct $\mathrm{p}$ waves, was considered as PoAF. Based on ECG findings, patients who did not develop PoAF during the postoperative hospitalization period constituted Group 1, and those who did were classified as Group 2.

Statistical analysis: SPSS 21.0 (IBM Statistical Package for Social Sciences Statistics Inc. version 21.0, Chicago, IL, USA) was used for data analysis. Mean and standard deviations were calculated for continuous and ordinal data. KolmogorovSmirnov test and Shapiro-Wilk test were used to evaluate the distribution of normality. Student's t test and Mann Whitney $\mathrm{U}$ tests were utilized to evaluate normally and non-normally distributed data, respectively. Frequency and percentage analysis were performed for nominal data and Chi Square test was used for its comparison. Multivariate binary logistic regression analysis was used to analyze the predictors of PoAF. A $P$ value less than .05 was considered statistically significant. ROC analysis was performed for the HATCH score, and NLR to predict postoperative AF and area under the curve (AUC) was calculated. Spearman correlation analysis 
Table 3. Operative/ Postoperative variables and laboratory values of the patients

\begin{tabular}{lccc}
\hline & $\begin{array}{c}\text { Group } 1 \\
(\mathrm{~N}=97) \\
(\text { mean } \pm \mathrm{SD})\end{array}$ & $\begin{array}{c}\text { Group } 2 \\
(\mathrm{~N}=26) \\
(\text { mean } \pm \mathrm{SD})\end{array}$ & $P$ \\
\hline White blood cell $\left(10^{3} / \mu \mathrm{L}\right)$ & $9.9 \pm 2.9$ & $9.6 \pm 3.1$ & .757 \\
Neutrophil $\left(10^{3} \mathrm{~mL}\right)$ & $4.9 \pm 1.4$ & $6.1 \pm 1.8$ & .076 \\
Lymphocyte $\left(10^{3} \mathrm{~mL}\right)$ & $2.1 \pm 0.9$ & $1.9 \pm 1.1$ & .345 \\
BUN (mg/dL) & $18.7 \pm 7.1$ & $19.2 \pm 8.9$ & .375 \\
Creatinine $(\mathrm{mg} / \mathrm{dL})$ & $1.2 \pm 0.8$ & $1.0 \pm 0.4$ & .556 \\
C Reactive protein $(\mathrm{mg} / \mathrm{dL})$ & $9.8 \pm 10.3$ & $9.1 \pm 12.6$ & .374 \\
Distan anostomosis number, $\mathrm{N}$ & $1.4 \pm 0.6$ & $1.6 \pm 0.5$ & .021 \\
NLR & $2.4 \pm 0.4$ & $2.9 \pm 0.5$ & .023 \\
Total chest tube drainage $(\mathrm{mL})$ & $596.4 \pm 249.8$ & $615.7 \pm 251.7$ & .794 \\
ICU stay (days) & $2.3 \pm 0.9$ & $3.1 \pm 1.1$ & $<.001$ \\
Total hospital stay $($ days $)$ & $6.4 \pm 1.1$ & $7.8 \pm 1.4$ & $<.001$ \\
\hline
\end{tabular}

BUN: blood urea nitrogen, NLR: neutrophil-to-lymphocyte ratio, ICU: intensive care unit

was used to perform the correlation analysis between the HATCH score and NLR.

\section{RESULTS}

Ninety-seven patients (69 males and 28 females) with a mean age of $54.4 \pm 11.1$ years constituted Group 1, and 26 patients (17 males and 9 females) with a mean age of $61 \pm$ 12.6 years constituted Group 2. There was no statistically significant difference between the 2 groups, in terms of diabetes mellitus (DM), hyperlipidemia (HL), hypertension (HT), gender distribution, and chronic obstructive pulmonary disease (COPD) rates. However, significant differences were observed, in terms of age and HATCH scores $(P=.025$, $P<.001$, respectively). Preoperative values and demographic data of the patients are presented in Table 2. (Table 2)

The blood values of the patients were shown in Table 3. There were no statistical differences between the 2 groups, in terms of blood parameters. However, NLR, number of distal anastomoses, intensive care unit (ICU) stay times, and total hospitalization times significantly were higher in Group 2 $(P=.021, P=.021, P<.001, P<.001$, respectively) (Table 3$)$.

We performed multivariate logistic regression analysis to identify factors affecting PoAF. While the HATCH score was identified as an independent predictor of AF development following OPCABG surgery (OR: 2.125, 95 \% CI: 1.296-3.482, $P=.003)$, number of distal anastomoses, NLR, and age were not. (Table 4)

In Roc Curve analysis, NLR was determined to predict PoAF at a cut-off level of 2.4 with a sensitivity of $65 \%$ and a specificity of $38 \%$ (area under the curve (AUC): 0.644, $95 \%$
Table 4. Multivariate Logistic Regression Analysis to Identify Factors Affecting PoAF

\begin{tabular}{lccc}
\hline Variables & Adjusted OR & $\% 95 \mathrm{Cl}$ & $P$ \\
\hline Age & 1.048 & $1.003-1.095$ & .073 \\
Distal anostomosis number & 1.944 & $0.910-4.154$ & .086 \\
NLR & 1.405 & $0.438-4.449$ & .567 \\
HATCH score & 2.125 & $1.296-3.482$ & .003 \\
\hline
\end{tabular}

NLR: neutrophil-tolymphocyte ratio

CI: $0.524-0.764, P=.024)$, and the HATCH score analysis revealed that a cut-off level of 2 predicted PoAF with a sensitivity of $69 \%$ and a specificity of $53 \%$ (AUC: $0.759,95 \%$ CI: $0.625-0.865, P<.001)$ (Figure 1).

\section{DISCUSSION}

In this study, we investigated the association of the HATCH score and NLR with PoAF development after OPCABG. In the literature, HATCH score was reported as an independent predictor for the development of PoAF after OPCABG. We also found a moderately positive correlation between NLR and the HATCH score, which includes inflammatory processes, such as heart failure and hypertension, as parameters.

AF development after coronary bypass graft surgery $(\mathrm{CABG})$ is an undesirable condition. In numerous studies, $\mathrm{AF}$ was reported to occur in more than half of the patients postoperatively [Bradley 2005; Alqahtani 2010]. Atrial fibrillation usually is detected within the first 5 postoperative days and is most common on the second day after surgery [Echahidi 2008]. Various factors, such as age-related fibrosis in the heart, low ejection fraction, lung and respiratory problems, increased left atrial diameter and body mass index, may play a role in the development of postoperative AF. Postoperative pericardial inflammation, electrolyte imbalances, changes in plasma fluid and catecholamine discharge also may lead to PoAF [Fuster 2001]. The higher incidence of PoAF in CABG operations with cardiopulmonary bypass may be caused by extracorporeal circulation [Bohatch Júnior 2015], which exposes the heart to external contact during pumping. Ectopic focal pulses may emerge from externally contacting pulmonary veins [Yadava 2016].

Low ejection fraction closely is related to $\mathrm{AF}$, and $\mathrm{EF}<$ $40 \%$ scores 2 points in the HATCH score evaluation. In cases of low EF, enlargement of the cavities of the heart, changes in myocardial structure, and structural remodeling may lead to the development of AF. In fact, AF can be detected in $25 \%$ of patients with heart failure [Lardizabal 2012]. The other valuable parameter, which scores 2 points, is TIA/stroke history. Although AF commonly is known to cause cerebrovascular events, our evaluation yielded different results. Patients with stroke or TIA may have had previous, undetected episodes 
of AF, which may have posed a risk for PoAF. In addition, $\mathrm{AF}$ development can be expected to increase in patients with mobility problems, due to cerebrovascular events. Patients with a history of cerebrovascular events may have atherosclerotic foci, and the extensiveness of atherosclerosis poses a risk for the development of AF [Heeringa 2007].

Age, HT and COPD, which constitute the other parameters of the HATCH score, are associated with increased risk of $\mathrm{AF}$, due to possible mobilization problems. In a study involving 36,258 Type 1 diabetes patients, HT and age were determined as risk factors for AF [Hallstrom 2019]. COPD also is a known predictor of AF [Li 2014], which makes the HATCH score a valuable PoAF predictor.

We found 2 studies investigating the relationship between the HATCH score and PoAF after CABG operations. In these studies, coronary operations were performed with CPB. In their study conducted on 284 patients, Emren et al detected PoAF in $25 \%$ of the patients and reported that the HATCH score significantly was higher in the PoAF group $(P<.001)$. After Roc analysis, a HATCH score of 2 or more was found to predict PoAF with $72 \%$ sensitivity and $75 \%$ specificity [Emren 2016]. Selvi et al reported a PoAF ratio of $28 \%$ in 369 patients. Performance of multivariate logistic regression analysis revealed that HATCH scores of 2 and above (sensitivity of $42 \%$ and specificity of $70 \%$ ) and high triglyceride levels were independent predictors of PoAF [Selvi 2017]. Our study was the first to investigate the association between HATCH scores and PoAF in patients, who underwent OPCABG. In multivariate analysis, high HATCH scores were found an independent predictor of PoAF (OR: 2.125, 95\% CI: $1.296-3.482, P=.003)$. ROC analysis proved that a HATCH score of 2 and above was predictive of PoAF with a sensitivity of $69 \%$ and specificity of $43 \%$.

The relationship between inflammatory biomarkers and cardiovascular disease has been demonstrated in many studies. Among these, NLR, which is a frequently researched biomarker, is cheap and easy to obtain. The inflammatory response following cardiac surgery mainly emerges from the cellular immune system [Laffey 2002]. Neutrophilia is induced by activation of cellular immunity by lymphocytes. NLR also increases, due to decreased lymphocyte count.

In a study investigating the prognostic significance of NLR in cardiac surgery, high NLR was associated with increased early-term complications and long-term mortality rates [Silberman 2018]. Research has shown that NLR can predict $\mathrm{AF}$ after open heart surgery [Weymann 2018]. However, it has been reported that the predictive value of NLR is more important in on-pump open heart surgery [Aldemir 2015]. Azab et al investigated the effect of NLR on long-term outcomes in OPCABG patients and demonstrated the association of high NLR with an increased incidence of PoAF [Azab 2013]. In our study, univariate analysis showed that NLR significantly was higher in patients with PoAF. However, in multivariate analysis, NLR was not determined as an independent predictor for PoAF. In Roc analysis, cut-off value was 2.4 with low sensitivity and specificity.

The aim of CABG operations without cardiopulmonary bypass is to prevent pump-induced inflammatory response, reduce complication rates, and shorten total duration of hospitalization. The use of cardiopulmonary bypass is an important risk factor for PoAF, which may be caused by possible ischemia due to cardioplegia and aortic cross-clamp as well as inflammatory response induced by the extracorporeal circulatory lines [Hashemzadeh 2013; Raja 2008]. Although PoAF-progressing effects of $\mathrm{CPB}$ have been identified, PoAF rates of on-pump and off-pump coronary bypass surgery were similar in the literature.

Accordingly, in a study by Lin et al it was shown that OPCABG surgery did not reduce PoAF incidence. The authors linked this to the increased preoperative use of betablockers in pumped surgery [Lin 2006]. In our study, preoperative beta blocker usage rates were similar between the 2 groups. In another study comparing the results of on-pump and off-pump coronary bypass operations, complications such as postoperative bleeding and stroke were found to have increased after on-pump surgery. In this study, PoAF rates in pumped and unpumped surgery were $19.7 \%$ and $13.4 \%$, respectively. There was no significant difference between the 2 groups [Bohatch Júnior 2015]. In conclusion, different results have been reported in the literature, regarding the development of PoAF in on-pump and off-pump surgery.

The relationship between NLR, which is an inflammatory marker, and chronic diseases has been proven by several studies. Inflammation plays a key role in the pathophysiology of various diseases such as hypertension, COPD, and heart failure. NLR values also may play a role in their prognosis [Liu 2015; Paliogiannis 2018]. Since these diseases are included in the HATCH score parameters, we hypothesized that a positive correlation may exist between NLR and the HATCH score. In the correlation analysis, we found a moderately positive correlation $(\mathrm{R}=0.427, P<.001)$.

\section{CONCLUSION}

$\mathrm{AF}$, which can occur after cardiac surgery is an undesirable condition that increases mortality and morbidity rates. It is particularly important for cardiac surgeons to identify possible risk factors for this condition. In the light of our study, HATCH scores of all patients may preoperatively be calculated so that preventive precautions are taken in high-risk patients. Further multicenter prospective studies with large patient series are needed for clearer results.

Study limitations: The most important limitation of our study was the small number of patients and its retrospective nature. Additionally, not all operations were performed by the same surgical team. Another important point is the diagnosis of PoAF in the follow-up of the patients. In our clinic, patients are followed in our intensive care unit with continuous ECG monitoring for the first 48 hours, then admitted to the inpatient clinic, where ECG is evaluated once a day and additionally in case of a complaint. Therefore, some PoAFs may have been omitted in patients, who did not report any complaints during inpatient follow-up. 


\section{REFERENCES}

Aldemir M, Bakı ED, Adalı F, et al. 2015. Comparison of neutrophil: lymphocyte ratios following coronary artery bypass surgery with or without cardiopulmonary bypass. Cardiovasc J Afr 26: 159-64.

Alqahtani AA. 2010. Atrial fibrillation post cardiac surgery trends toward management. Heart Views 11(2): 57-63.

Aviles RJ, Martin DO, Apperson-Hansen C, et al. 2003. Inflammation as a risk factor for atrial fibrillation. Circulation 108(24): 3006-3010.

Azab B, Shariff MA, Bachir R, et al. 2013. Elevated preoperative neutrophil/lymphocyte ratio as a predictor of increased long-term survival in minimal invasive coronary artery bypass surgery compared to sternotomy. J Cardiothorac Surg 8: 193.

Bohatch Júnior MS, Matkovski PD, Di Giovanni FJ, et al. 2015. Incidence of postoperative atrial fibrillation in patients undergoing on-pump and off-pump coronary artery bypass grafting. Braz J Cardiovasc Surg 30(3): 316-324.

Bradley D, Creswell L, Hogue CW Jr, et al. 2005. Pharmacologic prophylaxis: American College of Chest Physicians guidelines for the prevention and management of postoperative atrial fibrillation after cardiac surgery. Chest. 128(2 Suppl): 39S-47S.

Echahidi N, Pibarot P, O'Hara G, et al. 2008. Mechanisms, prevention, and treatment of atrial fibrillation after cardiac surgery. J Am Coll Cardiol 51: 793-801.

El-Chami MF, Kilgo P, Thourani V, et al. 2010. New-onset atrial fibrillation predicts long-term mortality after coronary artery bypass graft. J Am Coll Cardiol 55:1370-6.

Emren SV, Kocabaş U, Duygu H, et al. 2016. The role of HATCH score in predicting the success rate of sinus rhythm following electrical cardioversion of atrial fibrillation. Kardio Pol. 74(9): 978-984.

Fuster V, Rydén LE, Asinger RW, et al. 2001. ACC/AHA/ESC guidelines for the management of patients with atrial fibrillation. J Am Coll Cardiol 38:19.

Gibson PH, Croal BL, Cuthbertson BH et al. 2007. Preoperative neutrophil-lymphocyte ratio and outcome from coronary artery bypass grafting. Am Heart J 154(5): 995-1002.

Hallstrom S, Pivodic A, Rosengren A, et al. 2019. Risk Factors for Atrial Fibrillation in People With Type 1 Diabetes: An Observational Cohort Study of 36,258 Patients From the Swedish National Diabetes Registry. Diabetes Care 42(8):1530-1538.

Hashemzadeh K, Dehdilani M, Dehdilani M. 2013. Does Off-pump Coronary Artery Bypass Reduce the Prevalence of Atrial Fibrillation? J Cardiovasc Thorac Res 5(2):45-9.

Heeringa J, van der Kuip DAM, Hofman A, et al. 2007. Subclinical
Atherosclerosis and Risk of Atrial Fibrillation: The Rotterdam Study. Arch Intern Med 167(4):382-387.

Laffey JG, Boylan JF, Cheng DC. 2002. The systemic inflammatory response to cardiac surgery: Implications for the anesthesiologist. Anesthesiology 97: 215-252.

Lardizabal JA, Deedwania PC. 2012. Atrial fibrillation in heart failure. Med Clin North Am 96: 987-1000.

Li J, Agarwal SK, Alonso A, et al. 2014. Airflow obstruction, lung function, and incidence of atrial fibrillation: the atherosclerosis risk in communities (ARIC) study. Circulation 129: $971 \mathrm{e} 980$.

Lin WS, Liou JT, Yang SP, et al. 2006. Can off-pump coronary artery bypass graft surgery decrease the incidence of postoperative atrial fibrillation? Acta Cardiol Sin 22(4):205-11.

Liu X, Zhang Q, Wu H, et al. 2015. Blood neutrophil to lymphocyte ratio as a predictor of hypertension. Am J Hypertens 28(11):1339-46.

Miao DD, Zang XB, Zhang SL, et al. 2012. Predictive value of HATCH score on atrial fibrillation recurrence post radiofrequency catheter ablation. Zhonghua Xin Xue Guan Bing Za Zhi. 40(10):821-4.

Ozturk S, Kalyoncuoglu M, Sahin M. 2019. Comparison of SYNTAX Score I and SYNTAX Score II for Predicting Postoperative Atrial Fibrillation in Patients Undergoing Coronary Artery Bypass Graft Surgery. Heart Surg Forum 22(5): E319-E324.

Paliogiannis P, Fois AG, Sotgia S, et al. 2018. Neutrophil to lymphocyte ratio and clinical outcomes in COPD: recent evidence and future perspectives. Eur Respir Rev 27: 170113.

Papa A, Emdin M, Passino C, et al. 2008. Predictive value of elevated neutrophil-lymphocyte ratio on cardiac mortality in patients with stable coronary artery disease. Clin Chim Acta 395(1-2): 27-31.

Raja SG, Dreyfus GD. 2008. Current status of off-pump coronary artery bypass surgery. Asian Cardiovasc Thorac 16(2):164-78.

Selvi M, Gungor H, Zencir C, et al. 2017. A new predictor of atrial fibrillation after coronary artery bypass graft surgery: HATCH score. J Investig Med 66(3): 648-652.

Silberman S, Abu-Yunis U, Tauber R, et al. 2018. Neutrophil-lymphocyte ratio: prognostic impact in heart surgery. Early outcomes and late survival. Ann Torac Surg 105: 581-586.

Weymann A, Ali-Hasan-Al-Saegh S, Popov AF, et al. 2018. Hematologic indices as predictors of atrial fibrillation following isolated coronary artery bypass grafting, valvular surgery or combined procedures: A systematic review with meta-analysis. Kardiol Pol. 76(1): 107-118.

Yadava M, Hughay AB, Crawford TC. 2016. Postoperative Atrial Fibrillation Incidence, Mechanisms, and Clinical Correlates. Heart Failure Clin 12:299-308. 